\title{
Fixed points of automorphisms preserving the length of words in free solvable groups
}

\author{
Witold TOMASZEWSKI
}

\begin{abstract}
Let $\delta$ be an automorphism of prime order $p$ of the free group $F_{n}$. Suppose $\delta$ has no fixed points and preserves the length of words. By $\sigma:=\delta^{(m)}$ we denote the automorphism of the free solvable group $F_{n} / F_{n}^{(m)}$ induced by $\delta$. We show that every fixed point of $\sigma$ has the form $c c^{\sigma} \ldots c^{\sigma^{p-1}}$, where $c \in F_{n}^{(m-1)} / F_{n}^{(m)}$. This is a generalization of some known results, including the Macedońska-Solitar Theorem [10].
\end{abstract}

Mathematics Subject Classification (2010). 20F14, 20F16, $20 \mathrm{E} 36$.

Keywords. Automorphisms of groups, Solvable groups, Identities, Fixed points, Symmetric words.

1. Introduction. Motivation and the main result. If $G$ is an arbitrary group, then as usual $G^{(m)}$ is the $m$-th term of the derived series of $G$, that is $G^{(0)}=$ $G, G^{(1)}=G^{\prime}=[G, G]$, and for $m>1$ we have $G^{(m+1)}=\left[G^{(m)}, G^{(m)}\right]$. If $g, h$ are elements of a group, then $g^{h}=h^{-1} g h$ and $[g, h]=g^{-1} h^{-1} g h$. We denote the free group of finite or infinite rank by $F$ and by $F_{n}$ the free group of rank $n$, freely generated by $x_{1}, \ldots, x_{n}$. If $w \in F_{n}$, then $|w|$ is the length of $w$ in the variables $x_{1}, \ldots, x_{n}$. The group $F_{n} / F_{n}^{(m)}$ is the free solvable group of rank $n$, freely generated by $g_{i}=x_{i} F_{n}^{(m)}$ for $i \in\{1, \ldots, n\}$. Throughout this paper $I$ and $J$ are sets consisting of integers, and we assume that 1 belongs to both sets. For a family of groups $\left\{G_{i}\right\}_{i \in I}$, let $\prod_{i \in I} G_{i}$ and $\prod_{i \in I}^{*} G_{i}$ be respectively the direct and the free product of the groups of this family.

Let $\delta$ be an automorphism of $F_{n}$. We say that $\delta$ preserves the length of words in the variables $x_{1}, \ldots, x_{n}$ if for every word $w \in F_{n}$ we have $\left|w^{\delta}\right|=|w|$.

An automorphism $\delta$ preserves the length of words in the variables $x_{1}, \ldots, x_{n}$ if and only if there is a permutation $\sigma \in S_{n}$ and an $n$-tuple $\left(\varepsilon_{1}, \ldots, \varepsilon_{n}\right) \in$ $\{-1,1\}^{n}$ such that $x_{i}^{\delta}=x_{i^{\sigma}}^{\varepsilon_{i}}$. The set of all such automorphisms forms a subgroup $H$ of $\operatorname{Aut}\left(F_{n}\right)$. The subgroup $H$ has two natural subgroups. The first, 
$K$, is isomorphic to $Z_{2}^{n}$, the elementary abelian group of order $2^{n}$. We associate an $n$-tuple $\left(\varepsilon_{1}, \ldots, \varepsilon_{n}\right) \in Z_{2}^{n}$ with the automorphism $\xi \in K$ acting on free generators as follows $x_{i}^{\xi}=x_{i}^{(-1)^{\varepsilon_{i}}}$ for $i=1, \ldots, n$. The second subgroup, $L$, is isomorphic to the symmetric group $S_{n}$. If $\sigma \in S_{n}$, then the corresponding automorphism $\bar{\sigma} \in L$ acts on generators as follows: $x_{i}^{\bar{\sigma}}=x_{i^{\sigma}}$ for $i=1, \ldots, n$. We say that the automorphisms from $L$ permute the generators. It is easy to see that $K$ and $L$ are normal subgroups in $H$ and that, in fact, $H$ is isomorphic to the direct product $K \times L$ and to the direct product $Z_{2}^{n} \times S_{n}$. Hence every automorphism $\delta$ preserving the length of words can be uniquely decomposed as a product $\delta=\xi \sigma=\sigma \xi$, where $\xi \in K$ and $\sigma \in L$.

Let $\delta$ be an automorphism of $F_{n}$ without nontrivial fixed points and preserving the length of words. The automorphism $\delta$ induces an automorphism $\delta^{(m)}$ of the free solvable group $F_{n} / F_{n}^{(m)}$ by the action $\left(w F_{n}^{(m)}\right)^{\delta^{(m)}}=w^{\delta} F_{n}^{(m)}$. The aim of this work is to describe the subgroup of fixed points of $\delta^{(m)}$, that is

$$
S\left(\delta^{(m)}\right)=\left\{w \in F_{n} / F_{n}^{(m)}: w^{\delta^{(m)}}=w\right\} .
$$

Let $S^{(m)}(\delta)$ be the preimage of $S\left(\delta^{(m)}\right)$ in $F_{n}$, that is

$$
S^{(m)}(\delta)=\left\{w \in F_{n}: w^{-1} w^{\delta} \in F_{n}^{(m)}\right\} .
$$

This topic is connected with the notion of symmetric words in groups and the Marczewski-Płonka problem. We say that a word $w\left(x_{1}, \ldots, x_{n}\right)$ in $F_{n}$ is symmetric in a group $G$ if for any permutation $\alpha \in S_{n}, w\left(x_{1}, \ldots, x_{n}\right)=$ $w\left(x_{1^{\alpha}}, \ldots, x_{n^{\alpha}}\right)$ is the identity in $G$. If $w$ is an $n$-symmetric word in $G$, then the function $f: G^{n} \rightarrow G$ given by $f\left(g_{1}, \ldots, g_{n}\right)=w\left(g_{1}, \ldots, g_{n}\right)$ for every $n$-tuple $\left(g_{1}, \ldots, g_{n}\right) \in G^{n}$ is called a symmetric operation in $G$. Let $\mathcal{K}$ be a class of all groups $G$ such that the group operation $x y$ is a composition of symmetric operations. It is clear that all abelian groups are in $\mathcal{K}$. In 1967 Marczewski asked whether $\mathcal{K}$ consists only of abelian groups (see [11]). In 1970 Płonka gave in [12] an example of a non-abelian group which belongs to $\mathcal{K}$. But it is still an open question which groups belong to $\mathcal{K}$ (see [13]). In [15] Płonka described symmetric words in nilpotent groups of class $\leq 3$, and it follows from his description that non-abelian nilpotent groups do not belong to $\mathcal{K}$. In the series of papers [4-6] Hołubowski described symmetric words in free nilpotent groups of class 4 and 5, 2- and 3-symmetric words in free metabelian groups and in free metabelian nilpotent groups of any class. In [3] Gupta and Hołubowski found all 2-symmetric words in free nilpotentby-abelian groups and free centre-by-metabelian groups. In [10] Macedońska and Solitar characterized 2-symmetric words in free metabelian and solvable groups of derived length 3. I presented in [16] a description of 2-symmetric words in free solvable groups of any derived length, and in cooperation with Bagiński, I described in [1] fixed points of the automorphism cyclically permuting generators in free metabelian groups. In papers the [7-9,14,15], Płonka, Krstić, and Macedońska showed that if $G$ is a (free) nilpotent group, then the function $\delta_{n-1}^{n}\left(w\left(x_{1}, \ldots, x_{n}\right)\right)=w\left(x_{1}, \ldots, x_{n-1}, 1\right)$ is an isomorphism of the group of $n$-symmetric words onto the group of $(n-1)$-symmetric words. 
Let $G=F_{n} / V$ be a relatively free group, freely generated by elements $g_{1}=x_{1} V, \ldots, g_{n}=x_{n} V$. Then $w\left(x_{1}, \ldots, x_{n}\right) \in F_{n}$ is a symmetric word in $G$ if its image $w\left(g_{1}, \ldots, g_{n}\right)$ in $G$ is a fixed point for all automorphisms permuting the generators $g_{1}, \ldots, g_{n}$. Such automorphisms are induced by automorphisms permuting the generators $x_{1}, \ldots, x_{n}$ in $F_{n}$. In fact, a word $w\left(x_{1}, \ldots, x_{n}\right) \in F_{n}$ is the symmetric word in $G$ if and only if its image $w\left(g_{1}, \ldots, g_{n}\right)$ is a fixed point of two automorphisms. The one that interchanges $g_{1}$ and $g_{2}$ and acts identically on the rest of the generators and the other which cyclically permutes generators, i.e. acts on them as follows: $g_{1} \rightarrow g_{2} \rightarrow \cdots \rightarrow g_{n} \rightarrow g_{1}$.

The main result of this paper is the following:

Main Theorem. Let $\delta$ be a length preserving automorphism of $F_{n}$ of prime order $p$ and without nontrivial fixed points. Let $\delta^{(m)}$ be an automorphism of the free solvable group $G=F_{n} / F_{n}^{(m)}$ induced by $\delta$. Then every fixed point of $\sigma=\delta^{(m)}$ has the form $c c^{\sigma} \ldots c^{\sigma^{p-1}}$, where $c \in G^{(m-1)}=F_{n}^{(m-1)} / F_{n}^{(m)}$.

It is easy to see that every element of the form $c c^{\sigma} \cdots c^{\sigma^{p-1}}$, where $c \in$ $G^{(m-1)}=F_{n}^{(m-1)} / F_{n}^{(m)}$, is a fixed point for $\sigma=\delta^{(m)}$, but it is not obvious that only such elements are the fixed points.

The Main Theorem is a generalization of a result of Macedońska and Soli$\operatorname{tar}$ [10], who described the form of fixed points for automorphisms permuting generators in the 2-generator free metabelian group and free solvable group of derived length 3 . This was later generalized by the author [16] to include free solvable groups of any derived length. The Main Theorem is also a generalization of a result of Bagiński and the author [1] which gives a description of the fixed points of the automorphism cyclically permuting generators in free metabelian groups.

The Main Theorem gives the full description of 2-symmetric words in free solvable groups, which we formulate as follows:

Corollary 1. Let $w(x, y) \in F_{2}$ be a 2-symmetric word in any solvable group of derived length $m$. Then $w$ has the form $w=c(x, y) c(y, x) \xi$, where $c(x, y) \in$ $F_{2}^{(m-1)}$ and $\xi \in F_{2}^{(m)}$.

Proof. If $w(x, y)$ is a 2-symmetric word in any solvable group of the derived length $m$, then it is a 2 -symmetric word in $F_{2} / F_{2}^{(m)}$. So by the Main Theorem $w$ has the required form.

The next result follows directly from the Main Theorem

Corollary 2. The free, solvable, non-abelian groups of finite rank do not belong to $\mathcal{K}$.

Proof. Let $G=F_{n} / F_{n}^{(m)}$ be a free, non-abelian, solvable group, freely generated by $g_{1}, \ldots, g_{n}$. Let $\alpha$ be the automorphism cyclically permuting the generators of the free group $F_{n}$ that is acting on generators of the free group as follows: $x_{1} \rightarrow x_{2} \rightarrow \cdots \rightarrow x_{n} \rightarrow x_{1}$. There exists a positive integer $k$ such that $\beta=\alpha^{k}$ has no nontrivial fixed points in $F_{n}$ and has prime order $p$. If $w \in F_{n}$ is an $n$-symmetric word in $G$, then its image $\bar{w}$ in $G$ has to be a 
fixed point of $\bar{\beta}$, so by the Main Theorem, $\bar{w}$ belongs to $G^{(m-1)}$, and since $G$ is non-abelian, we have $m>1$, so $\bar{w}$ belongs to $G^{\prime}$. If $x y$ were a composition of symmetric operations, it would belong to $G^{\prime}$. However, this would be impossible.

2. Proofs. Throughout this section we assume that $\delta$ is an automorphism of $F_{n}$ preserving the length of words and that $\delta$ has no nontrivial fixed points in $F_{n}$. The proof of the Main Theorem is based on Dyer-Scott's Theorem on automorphisms of prime order in a free group [2].

Proposition 1. Let $\delta$ be an automorphism of $F_{n}$ of order $k$ that has no nontrivial fixed points and preserves the length of words in the variables $x_{1}, \ldots, x_{n}$. Let $w$ and $c$ be elements of $F_{n}$.

(i) The equation $c c^{\delta} \ldots c^{\delta^{k-1}}=1$ holds if and only if there exists $u \in F_{n}$ such that $c=u^{-1} x^{\varepsilon} u^{\delta}$, where $x \in\left\{x_{1}, \ldots, x_{n}\right\}$ is such that $x^{\delta}=x^{-1}$ and $\varepsilon \in\{-1,0,1\}$. Moreover, if $k$ is odd, then $\varepsilon=0$ and $c=u^{-1} u^{\delta}$.

(ii) There are no $a \in F_{n}$ and $x \in\left\{x_{1}^{ \pm 1}, \ldots, x_{n}^{ \pm 1}\right\}$ which satisfy $x^{\delta}=x^{-1}$ and $a^{\delta}=x a x$.

(iii) If $w^{\delta}=c^{-1} w c$ and $c c^{\delta} \ldots c^{\delta^{k-1}}=1$, then $w=1$.

Proof. (i) Note that if $k$ is odd, then there is no $x \in\left\{x_{1}, \ldots, x_{n}\right\}$ such that $x^{\delta}=x^{-1}$, so in this case $\varepsilon=0$. If $c=u^{-1} x^{\varepsilon} u^{\delta}$, then

$$
\begin{aligned}
& c c^{\delta} \ldots c^{\delta^{p-1}}=u^{-1} x^{\varepsilon} u^{\delta}\left(u^{-1} x^{\varepsilon} u^{\delta}\right)^{\delta}\left(u^{-1} x^{\varepsilon} u^{\delta}\right)^{\delta^{2}} \ldots\left(u^{-1} x^{\varepsilon} u^{\delta}\right)^{\delta^{k-1}} \\
& \quad=u^{-1} x^{\varepsilon} u^{\delta} u^{-\delta} x^{-\varepsilon} u^{\delta^{2}} u^{-\delta^{2}} x^{\varepsilon} u^{\delta^{3}} \ldots u^{-\delta^{k-1}} x^{(-1)^{k} \varepsilon} u^{\delta^{k}}=u^{-1} u^{\delta^{k}}=u^{-1} u=1 .
\end{aligned}
$$

For the converse, we use induction on the length of a word $c$. If $c$ has the length 1 , then $c \in\left\{x_{1}^{ \pm 1}, \ldots, x_{n}^{ \pm 1}\right\}$, and it satisfies $c c^{\delta} \ldots c^{\delta^{k-1}}=1$ only if $c^{\delta}=c^{-1}$. If $|c|=2$, then $c=x_{i}^{\varepsilon_{1}} x_{j}^{\varepsilon_{2}}$, where $\varepsilon_{1}, \varepsilon_{2}= \pm 1$. For the word $c c^{\delta} \ldots c^{\delta^{k-1}}$ to cancel, we need $\left(x_{i}^{\varepsilon_{1}}\right)^{\delta}=x_{j}^{-\varepsilon_{2}}$ and then $c=u^{-1} u^{\delta}$ where $u=$ $x_{i}^{-\varepsilon_{1}}$. Let the statement be true for every word of length less than $|c|>2$. Then $c=x_{i}^{\varepsilon_{1}} c_{1} x_{j}^{\varepsilon_{2}}$ and $c c^{\delta} \ldots c^{\delta^{k-1}}=x_{i}^{\varepsilon_{1}} c_{1} x_{j}^{\varepsilon_{2}}\left(x_{i}^{\varepsilon_{1}} c_{1} x_{j}^{\varepsilon_{2}}\right)^{\delta} \ldots\left(x_{i}^{\varepsilon_{1}} c_{1} x_{j}^{\varepsilon_{2}}\right)^{\delta^{k-1}}$.

For the word $c c^{\delta} \ldots c^{\delta^{k-1}}$ to cancel, we need again $\left(x_{i}^{\varepsilon_{1}}\right)^{\delta}=x_{j}^{-\varepsilon_{2}}$ and then $1=c c^{\delta} \ldots c^{\delta^{k-1}}=x_{i}^{\varepsilon_{1}} c_{1} c_{1}^{\delta} \ldots c_{1}^{\delta^{k-1}} x_{i}^{-\varepsilon_{1}}$. So $c_{1}$ satisfies the equation $c_{1} c_{1}^{\delta} \ldots c_{1}^{\delta^{k-1}}=1$, and by the inductional assumption it has the form $c_{1}=u_{1}^{-1} x^{\varepsilon} u_{1}^{\delta}$. Hence $c$ has the form $c=u^{-1} x^{\varepsilon} u^{\delta}$, where $u=u_{1} x_{i}^{-\varepsilon_{1}}$.

(ii) Assume that $a^{\delta}=x a x$, where $x$ is a generator or its inverse, satisfying $x^{\delta}=x^{-1}$. We have $|a|=\left|a^{\delta}\right|=|x a x|$, so we must have $a=x^{-1} b$ or $a=b x^{-1}$ for some $b$ such that $|b|=|a|-1$. Consider the first case, the second is analogous. We have $a^{\delta}=\left(x^{-1} b\right)^{\delta}=x a x=b x$ and hence $x b^{\delta}=b x$. So, $b^{\delta}=x^{-1} b x$. Again $|b|=\left|x^{-1} b x\right|$, which means that $b=u x^{-1}$ or $b=x u$ for some $u$ such that $|u|=|b|-1=|a|-2$. The case $b=u x^{-1}$ is impossible because then $a^{\delta}=b x=u$ and $|a|=\left|a^{\delta}\right|=|u|=|a|-2$. The second case is also impossible because $a^{\delta}=x a x=b x=x u x$. It follows from this that $a=u$, but $a$ and $u$ have different lengths.

(iii) We can assume that a word satisfying these assumptions is cyclically reduced. If not, then we can consider a cyclically reduced word $w^{v}$, for which 


$$
\left(w^{v}\right)^{\delta}=\left(w^{\delta}\right)^{\delta}=v^{-\delta} c^{-1} w c v^{\delta}=\left(v^{-\delta} c^{-1} v\right) w^{v}\left(v^{-1} c v^{\delta}\right),
$$

and we have

$$
\begin{aligned}
& \left(v^{-1} c v^{\delta}\right)\left(v^{-1} c v^{\delta}\right)^{\delta}\left(v^{-1} c v^{\delta}\right)^{\delta^{2}} \ldots\left(v^{-1} c v^{\delta}\right)^{\delta^{k-2}}\left(v^{-1} c v^{\delta}\right)^{\delta^{k-1}} \\
& =v^{-1} c v^{\delta} v^{-\delta} c^{\delta} v^{\delta^{2}} v^{-\delta^{2}} c^{\delta^{2}} v^{\delta^{3}} \ldots v^{-\delta^{k-2}} c^{\delta^{k-2}} v^{\delta^{k-1}} v^{-\delta^{k-1}} c^{\delta^{k-1}} v^{\delta^{k}} \\
& =v^{-1} \underbrace{c c^{\delta} \ldots c^{\delta^{k-2}} c^{\delta^{k-1}}}_{=1} v^{\delta^{k}}=v^{-1} v=1 .
\end{aligned}
$$

So $w^{v}$ also satisfies the assumptions of the Proposition for $c_{1}=v^{-\delta} c^{-1} v$. Moreover, since $\delta$ does not change the length of words, we have $|w|=\left|w^{c}\right|$. So there exists $v$ such that $w=c v$ or $w=v c^{-1}$. It is enough to consider the first equality because the reasoning for the second is analogous. If $w=c v$, then $(c v)^{\delta}=(c v)^{c}=v c$. Hence, $v c=c^{\delta} v^{\delta}$ and $v^{\delta}=c^{-\delta} v c$. Using (i) we have that $c=u^{-1} x^{\varepsilon} u^{\delta}$, and so $v^{\delta}=\left(u^{-1} x^{\varepsilon} u^{\delta}\right)^{-\delta} v u^{-1} x^{\varepsilon} u^{\delta}=$ $u^{-\delta^{2}} x^{\varepsilon} u^{\delta} v u^{-1} x^{\varepsilon} u^{\delta}$. So we get:

$$
x^{\varepsilon} u^{\delta} v u^{-1} x^{\varepsilon}=u^{\delta^{2}} v^{\delta} u^{-\delta}=\left(u^{\delta} v u^{-1}\right)^{\delta} .
$$

If $\varepsilon \neq 0$, then for $a=u^{\delta} v u^{-1}$ we get $a^{\delta}=x a x$, which by (ii) is impossible. If $\varepsilon=0$, then $u^{\delta} v u^{-1}$ is a fixed point of $\delta$. But $\delta$ has no nontrivial fixed points, so $u^{\delta} v u^{-1}=1$. Hence $v=u^{-\delta} u=\left(u^{-1} u^{\delta}\right)^{-1}=c^{-1}$ and $w=c v=c c^{-1}=1$, as required.

Lemma 1. Let $F_{n}$ be a free group, freely generated by $x_{1}, \ldots, x_{n}$, and let $\delta$ be an automorphism of $F_{n}$ of order $p$ which has no nontrivial fixed points and which does not change the length of words. Then for every positive integer $m$, the subgroup $F_{n}^{(m)}$ is a free product $\prod_{i \in I}^{*}\left\langle a_{i 1}, \ldots, a_{i p}\right\rangle * \prod_{j \in J}^{*}\left\langle c_{j 1}, \ldots, c_{j(p-1)}\right\rangle$, where $I, J$ are sets of positive integers, and for all $i \in I$ and $j \in J: a_{i 1}^{\delta}=$ $a_{i 2}, a_{i 2}^{\delta}=a_{i 3}, \ldots, a_{i(p-1)}^{\delta}=a_{i p}, a_{i p}^{\delta}=a_{i 1}, c_{j 1}^{\delta}=c_{j 2}, c_{j 2}^{\delta}=c_{j 3}, \ldots, c_{j(p-1)}^{\delta}=$ $c_{j(p-1)}^{-1} c_{j(p-2)}^{-1} \ldots c_{j 1}^{-1}$.

Proof. Let $\delta_{m}$ be a restriction of $\delta$ to $F_{n}^{(m)}$. Then $\delta_{m}$ is an automorphism of prime order $p$ of $F_{n}^{(m)}$. Dyer and Scott proved [2, Theorem 3] that then $F_{n}^{(m)}$ is a free product $F^{\left\langle\delta_{m}\right\rangle} * \prod_{i \in I}^{*} F_{i} * \prod_{j \in J}^{*} F_{j}$, where $F^{\left\langle\delta_{m}\right\rangle}$ is a subgroup of fixed points of $\delta_{m}, F_{i}=\left\langle a_{i 1}, \ldots, a_{i p}\right\rangle, a_{i 1}^{\delta}=a_{i 2}, a_{i 2}^{\delta}=a_{i 3}, \ldots, a_{i(p-1)}^{\delta}=a_{i p}, a_{i p}^{\delta}=$ $a_{i_{1}}, F_{j}=\left\langle c_{j 1}, \ldots, c_{j(p-1)}, w_{k, j}, k \in J_{j}\right\rangle$, where $c_{j 1}^{\delta}=c_{j 2}, c_{j 2}^{\delta}=c_{j 3}, \ldots, c_{j(p-1)}^{\delta}=$ $c_{j(p-1)}^{-1} c_{j(p-2)}^{-1} \ldots c_{j 1}^{-1}$, and for every $k \in J_{j}$ we have $w_{k, j}^{\delta}=c_{j 1}^{-1} w_{k, j} c_{j 1}$. It is clear that $F^{\left\langle\delta_{m}\right\rangle}$ is trivial ( $\delta$ has no nontrivial fixed points and neither does $\left.\delta_{m}\right)$. The word $c_{j 1}$ satisfies the equation

$$
c_{j 1} c_{j 1}^{\delta} \ldots c_{j 1}^{\delta^{p-1}}=c_{j 1} c_{j 2} \ldots c_{j(p-1)} c_{j(p-1)}^{-1} c_{j(p-2)}^{-1} \ldots c_{j 1}^{-1}=1,
$$

so by Proposition 1 (iii) the words $w_{k, j}$ have to be trivial, so $F_{j}=\left\langle c_{j 1}, \ldots\right.$ $\left.c_{j(p-1)}\right\rangle$, and the statement of the Lemma follows. 
The similar basis as in Lemma 1 for automorphism interchanging generators in $F_{2}$ was constructed by the author in [16]. This construction uses the special basis in free groups of countable rank described in [16] or in [17]. But the author cannot use this technique for automorphisms permuting generators in free groups of rank greater than 2 .

Lemma 2. Let $A=\prod_{i \in I}\left\langle A_{i}\right\rangle \times \prod_{j \in J}\left\langle C_{j}\right\rangle$ be a free abelian group, freely generated by the union of sets $A_{i}=\left\{\alpha_{i 1}, \ldots, \alpha_{i p}\right\}$ and $C_{j}=\left\{\gamma_{j 1}, \ldots, \gamma_{j(p-1)}\right\}$ for $i \in I, j \in J$, and let $\varphi$ be an automorphism of $A$ acting on subgroups $\left\langle A_{i}\right\rangle$ and $\left\langle C_{j}\right\rangle$ as follows: $\alpha_{i 1}^{\varphi}=\alpha_{i 2}, \alpha_{i 2}^{\varphi}=\alpha_{i 3}, \ldots, \alpha_{i(p-1)}^{\varphi}=\alpha_{i p}, \alpha_{i p}^{\varphi}=\alpha_{i 1}, \gamma_{j 1}^{\varphi}=$ $\gamma_{j 2}, \gamma_{j 2}^{\varphi}=\gamma_{j 3}, \ldots, \gamma_{j(p-1)}^{\varphi}=\gamma_{j(p-1)}^{-1} \gamma_{j(p-2)}^{-1} \cdots \gamma_{i 1}^{-1}$. Then every fixed point a of $\varphi$ has the form $a=c c^{\varphi} \ldots c^{\varphi^{p-1}}$, where $c \in \prod_{i \in I} A_{i}$.

Proof. It is easy to see that the statement is true if $a \in A_{i}$ for any $i \in I$ and that $\varphi$ has no nontrivial fixed points in $C_{j}$ for any $j \in J$. The Lemma follows since $\varphi$ acts independently on direct summands of $A$.

Remark 1. Without loss of generality, we can assume that every fixed point of $\varphi$ satisfying the assumptions of the previous lemma has the form $c c^{\varphi} \ldots c^{\varphi^{p-1}}$, where $c=\alpha_{11}^{d_{1}} \ldots \alpha_{k 1}^{d_{k}}$ for some $k$, where $a_{i 1} \in A_{i}$.

Remark 2. It follows from Lemma 1 that for every $m$, a group $F_{n}^{(m)} / F_{n}^{(m+1)}$ has a basis such that the automorphism $\varphi=\bar{\delta}_{m}$ satisfies the assumptions of Lemma 2 , where $\bar{\delta}_{m}$ is a restriction of $\bar{\delta}$ onto $F_{n}^{(m)} / F_{k}^{(m+1)}$. We will use this remark both for $F_{n}^{(m)} / F_{n}^{(m+1)}$ and

$$
F_{n}^{(m-1)} / F_{n}^{(m)} \simeq\left(F_{n}^{(m-1)} / F_{n}^{(m+1)}\right) /\left(F_{n}^{(m)} / F_{n}^{(m+1)}\right) .
$$

Lemma 3. For every integer $m>0$ we have:

(i) $S^{(m+1)}(\delta) \subseteq S^{(m)}(\delta)$

(ii) $F_{n}^{(m)} \subseteq S^{(m)}(\delta) \subseteq F_{n}^{(m-1)}$,

(iii) $S^{(m+1)}(\delta)$ is a normal subgroup of $S^{(m)}(\delta)$.

Proof. Let us be reminded that

$$
S^{(m)}(\delta)=\left\{w \in F_{n}: w^{-1} w^{\delta} \in F_{n}^{(m)}\right\} .
$$

(i) If $w$ belongs to $S^{(m+1)}(\delta)$, then $w^{-1} w^{\delta} \in F_{n}^{(m+1)} \subseteq F_{n}^{(m)}$, so $w \in S^{(m)}(\delta)$.

(ii) The inclusion $F_{n}^{(m)} \subseteq S^{(m)}(\delta)$ is trivial, so we shall prove that $S^{(m)}(\delta) \subseteq$ $F_{n}^{(m-1)}$. We use an induction on $m$. For $m=1$ the situation is clear. Now suppose that the statement is true for some $m>0$. By part (i) and the induction hypothesis $S^{(m+1)}(\delta) \subseteq S^{(m)}(\delta) \subseteq F_{n}^{(m-1)}$. Let $u \in S^{(m+1)}(\delta)$. We know that $u \in S^{(m)}(\delta) \cap F_{n}^{(m-1)}$, and we want to show that $u \in F_{n}^{(m)}$. By Lemmas 1 and 2 we have that $u=a a^{\delta} \ldots a^{\delta^{p-1}} z$ with

$$
a=a_{1}^{d_{1}} \ldots a_{k}^{d_{k}},
$$


where $a_{1}, a_{1}^{\delta}, \ldots, a_{1}^{\delta^{p-1}}, \ldots, a_{k}, a_{k}^{\delta}, \ldots, a_{k}^{\delta^{p-1}}$ are among the free generators for $F_{n}^{(m-1)}$ and $z \in F_{n}^{(m)}$. Let $\phi_{i}$ be an endomorphism of $F_{n}^{(m-1)}$ that fixes the generators $a_{i}, a_{i}^{\delta}, \ldots, a_{i}^{\delta^{p-1}}$ and maps all other generators to 1 . Clearly, $\phi_{i}$ commutes with the action of $\delta$ on $F_{n}^{(m-1)}$ and thus

$$
v_{i}=u^{\phi_{i}}=b b^{\delta} \ldots b^{\delta^{p-1}} z^{\phi_{i}}
$$

is also in $S^{(m+1)}(\delta)$, where $b=a_{i}^{d_{i}}$. Then $\bar{v}_{i}$, the image of $v_{i}$ in $F_{n}^{(m-1)} / F_{n}^{(m+1)}$, is a fixed point of an automorphism cyclically permuting the generators of the free metabelian group $\left\langle\bar{a}_{i}, \bar{a}_{i}^{\delta}, \ldots, \bar{a}_{i}^{\delta^{p-1}}\right\rangle$. It follows from [1, Theorem 3] that $\bar{v}_{i}$ belongs to $\left\langle\bar{a}_{i}, \bar{a}_{i}^{\delta}, \ldots, \bar{a}_{i}^{\delta^{p-1}}\right\rangle^{\prime}$, which means that $d_{i}=0$. This shows that $d_{1}=d_{2}=\cdots=d_{k}=0$ and thus $u \in F_{n}^{(m)}$.

(iii) Let $w \in S^{(m+1)}(\delta)$ and $v \in S^{(m)}(\delta)$. By assumption $v^{-1} v^{\delta} \in F_{n}^{(m)}$ and $w^{-1} w^{\delta} \in F_{n}^{(m+1)}$. By (ii) we know that $w \in F_{n}^{(m)}$, and thus $w$ commutes with $v^{-1} v^{\delta}$ modulo $F_{n}^{(m+1)}$. Thus, modulo $F_{n}^{(m+1)}$, we have

$$
\begin{aligned}
& \quad\left(w^{v^{-1}}\right)^{-1}\left(w^{v^{-1}}\right)^{\delta}=v w^{-1} v^{-1} v^{\delta} w^{\delta}\left(v^{\delta}\right)^{-1}=v v^{-1} v^{\delta} w^{-1} w^{\delta}\left(v^{\delta}\right)^{-1} \\
& \quad=v^{\delta}\left(v^{\delta}\right)^{-1}=1, \\
& \text { so } w^{v^{-1}}=v w v^{-1} \in S^{(m+1)}(\delta) .
\end{aligned}
$$

Proof of the Main Theorem. If $u$ is a fixed point of $\delta^{(m+1)}$ in $F_{n} / F_{n}^{(m)}$, then by Lemma 3 (ii) $u$ belongs to $F_{n}^{(m-1)} / F_{n}^{(m)}$, which is a free abelian group. By Remark 2, if $\varphi$ is a restriction of $\delta^{(m+1)}$ to $F_{n}^{(m-1)} / F_{n}^{(m)}$, then $\varphi$ satisfies the assumptions of Lemma 2. So $u$ has the required form.

There are many questions connected with the topic of this paper to which the author so far has no answers.

For example, is it true that $S^{(m)}(\delta) / S^{(m+1)}(\delta)$ is a free metabelian group?

Acknowledgements. The author wishes to thank Vitaliy Sushchansky for his critical reading of this text and for many helpful remarks, due to which this paper is of a more general form than its first version. The author would also like to thank Czesław Bagiński who read the first version of this text and offered many valuable suggestions.

Open Access. This article is distributed under the terms of the Creative Commons Attribution License which permits any use, distribution, and reproduction in any medium, provided the original author(s) and the source are credited.

\section{References}

[1] Cz. Bagiński and W. Tomaszewski, Automorphisms of prime order of free metabelian groups, Comm. Algebra 30 (2002), 4985-4996.

[2] J.L. Dyer And G.P. Scott, Periodic automorphisms of free groups, Comm. Algebra 3 (1975), 195-201.

[3] C.K. Gupta and W. HoŁubowski, On 2-symmetric words for groups, Arch. Math. (Basel) 73 (1999), 327-331. 
[4] W. Hoєubowski, Symmetric words in metabelian groups, Comm. in Algebra 23 (1995), 5161-5167.

[5] W. HoŁubowski, Symmetric words in a free nilpotent group of class 5, Groups St. Andrews 1997 in Bath, I, 363-367.

[6] W. HoŁubowski, Symmetric words in free nilpotent groups of class 4, Publ. Math. Debrecen 57 (2000), 411-419.

[7] S. Krstić, On symmetric words in nilpotent groups, Publ. Inst. Math. (Beograd) (N.S.) 27(41) (1980), 139-142.

[8] S. A. KRstić, On symmetric words in nilpotent groups, Algebraic Conference (Skopje, 1980), pp. 59-60, Posebni Izdanija, 2(19), Univ. "Kiril et Metodij", Skopje, 1980.

[9] O. Macedońska-Nosalska, On symmetric words in nilpotent groups, Fund. Math. 120 (1984), 119-125.

[10] O. Macedońska And D. Solitar , On binary $\delta$-invariant words in nilpotent groups, AMS Contemporary Math. 169 (1994), 431-449.

[11] E. Marczewski, Problem P 619, Colloq. Math. 17 (1967), 369.

[12] E. PŁonka, Symmetric operations in groups, Colloq. Math. 21 (1970), 179-186.

[13] E. PŁonka, Problem P 684, Colloq. Math. 21 (1970), 339.

[14] E. PŁonka, On symmetric words in free nilpotent groups, Bull. Acad. Polon. Sci. Sér. Sci. Math. Astronom. Phys. 18 (1970), 427-429.

[15] E. PŁonkA, Symmetric words in nilpotent groups of class $\leq 3$, Fundamenta Math. XCVII (1977), 95-103.

[16] W. Tomaszewski, Automorphisms permuting generators in groups and their fixed points, Ph.D. Thesis (in Polish), University of Silesia, Katowice 1999.

[17] W. Tomaszewski, A Basis of Bachmuth Type in the Commutator Subgroup of a Free Group, Canad. Math. Bull., 46 (2003), 299-303.

Witold TOMASzEWski

Institute of Mathematics,

Silesian University of Technology,

Kaszubska 23, 44-100 Gliwice, Poland

e-mail: Witold.Tomaszewski@polsl.pl

Received: 1 May 2012 